\title{
A digital holography technique for generating beams with arbitrary polarization and shape
}

\author{
David Maluenda $^{a}$, Ignasi Juvells $^{a}$, Rosario Martínez-Herrero ${ }^{b}$, Artur Carnicer $^{a}$ \\ ${ }^{a}$ Departament de Física Aplicada i Òptica, Universitat de Barcelona (UB), Martí i Franquès \\ 1, 08028 Barcelona (Spain); ${ }^{b}$ Departamento de Óptica, Facultad de Ciencias Físicas, \\ Universidad Complutense de Madrid, 28040 Madrid (Spain)
}

\begin{abstract}
A method for generating beams with arbitrary polarization and shape is proposed. Our design requires the use of a Mach-Zehnder set-up combined with translucent liquid crystal displays in each arm of the interferometer; in this way, independent manipulation of each transverse beam components is possible. The target of this communication is to develop a numerical procedure for calculating the holograms required for dynamically encode any amplitude value and polarization state in each point of the wavefront. Several examples demonstrating the capabilities of the method are provided.
\end{abstract}

Keywords: spatially-variant polarization, computer-generated holography, spatial light modulators

\section{INTRODUCTION}

Recently, the interest for arbitrary spatially-variant polarized beams (ASPB) has increased significantly due to their special properties compared to homogeneously polarized beams thus enhancing the functionality of optical systems. ${ }^{1}$ The generation process of ASPB using spatial light modulators (SLM), which act as controlled optical phase retarders or amplitude modulators, is still a challenging task..$^{2-5}$ The aim of this communication is to develop an optical system able to encode and reconfigure both the polarization and the shape of the beam.

Our approach is based on a Mach-Zehnder interferometric configuration combined with a translucent phasemostly modulator in each path of the interferometer. ${ }^{6,7}$ To separate horizontal and vertical component from each other the oscillation direction of one of the beams is rotated $90^{\circ}$. The transverse components of the incident light beam are processed independently and modified by means of specifically designed holograms. Subsequently, the components are recombined and led into the on-axis reconstruction system consisting of a $4 \mathrm{f}$ lens arrangement. In particular, the holograms have been calculated by means of an algorithm derived from Arrizon's method to encode complex optical signals using phase-mostly SLMs. ${ }^{8-10}$ This approach entitles us to encode any polarization state in each point of the wavefront even when the displays used are not able to modulate the phase from 0 to $2 \pi$ and present amplitude coupling. Additionally, the amplitude of the wavefront may also be modeled so as to obtain a particular shape.

The numerical procedure to generate the holograms is discussed and illustrative examples with complex polarization configurations and shapes are also considered. The paper is organized as follows: in section 2 we present the optical setup required to generate ASPB and in section 3 the algorithm for calculating complexvalued phase-mostly holograms is introduced. Some computational results are presented in section 4 whereas in section 5 , the main conclusions are summarized.

Corresponding author: A.C.: E-mail: artur.carnicer@ub.edu 


\section{OPTICAL SETUP}

Figure 1 presents the proposed experimental setup. A polarized laser beam is split up into two beams by means of a polarizing beam splitter $\mathrm{PBS}_{1}$, being the incident polarization direction set at $45^{0}$ with respect to the $x$ axis. Let $\mathbf{E}(x, y)$ a cylindrical ASPB whose electric field vibrates within the plane $x y$, normal to the propagation direction $z$. This beam is described by

$$
\mathbf{E}(x, y)=E_{x}(x, y) \mathbf{i}+E_{y}(x, y) \mathbf{j},
$$

where

$$
\begin{aligned}
& E_{x}(x, y)=E_{x 0}(x, y) \exp \left(i \phi_{\mathrm{x}}(x, y)\right) \text { and } \\
& E_{y}(x, y)=E_{y 0}(x, y) \exp \left(i \phi_{\mathrm{y}}(x, y)\right)
\end{aligned}
$$

are the complex amplitudes in every point $(x, y)$ and $\phi_{x}(x, y)$ and $\phi_{y}(x, y)$ are the corresponding phase shifts. Notice that the amplitude and the polarization direction of $\mathbb{E}(x, y)$ can be different in every point. The total phase difference is $\phi(x, y)=\phi_{x}(x, y)-\phi_{y}(x, y)$.

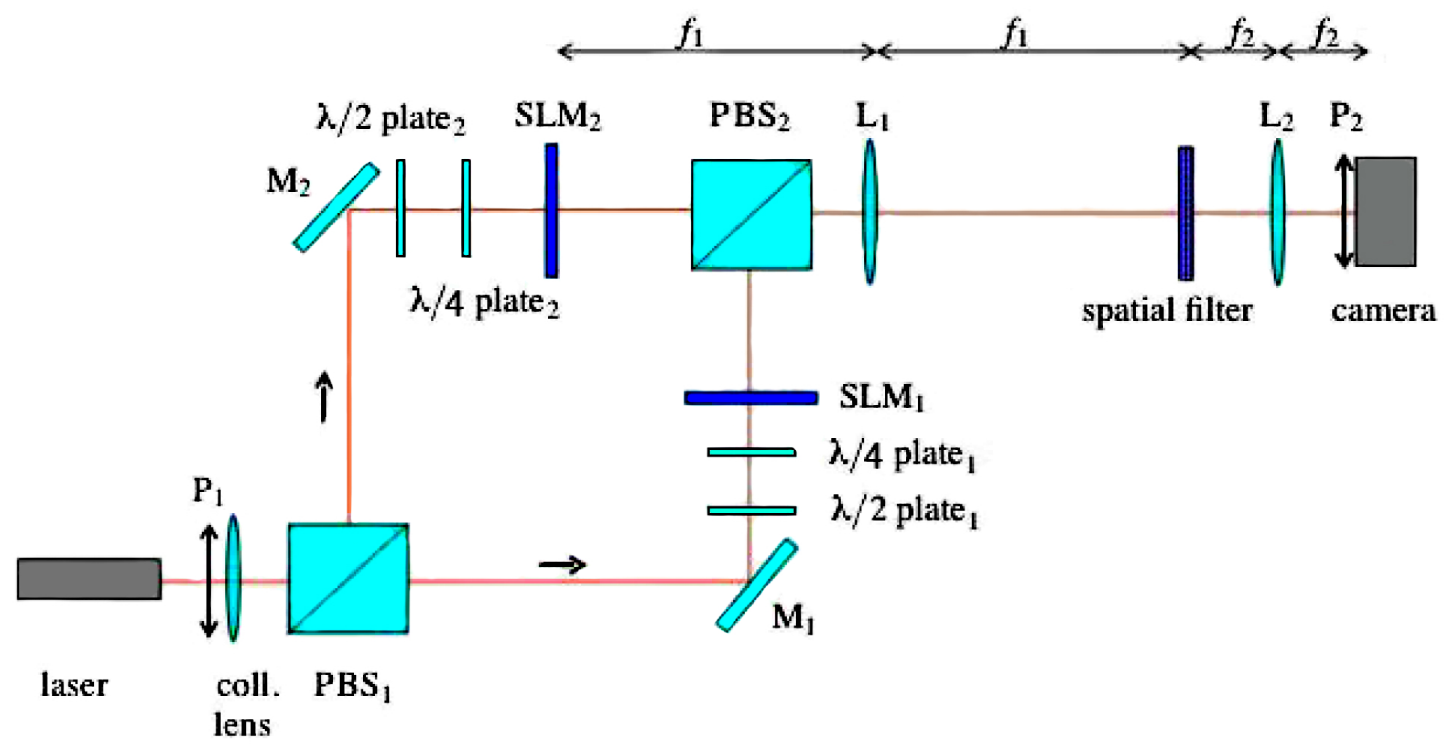

Figure 1: Sketch of the experimental setup.

A collimating lens aligns the entering unpolarized HeNe laser beam to propagate parallel along the optical path. The beam passes trough linear polarizer $\mathrm{P}_{1}$ (set at $45^{\circ}$ with respect to the $x$ direction) and a polarizing beam splitter $\mathrm{PBS}_{1}$. Reflected by mirrors $\mathrm{M}_{1}$ or $\mathrm{M}_{2}$, the split beams pass through different wave plates which rotate the oscillating plane and set the modulator in order to achieve the expected response of the SLM. Afterward, light passes through modulators $\mathrm{SLM}_{1}$ or $\mathrm{SLM}_{2}$. The displays used are two translucent twisted nematic Holoeye HEO $0017^{11}$ with a resolution of $1024 \times 768$ pixels and $32 \mu \mathrm{m}$ of pixel pitch. Then, light is recombined by means of the second polarizing beam splitter $\mathrm{PBS}_{2}$ and led into the on-axis reconstruction system consisting of a $4 f$-Fourier lens system with $\mathrm{L}_{1}$ with focal length $f_{1}$ and $\mathrm{L}_{2}$ with focal length $f_{2}$, respectively. Notice that a spatial filter in the back focal plane of $\mathrm{L}_{1}$ is required for removing non-required higher-order diffracted terms generated by the hologram. Finally, the induced polarization is evaluated by analyzer $\mathrm{P}_{2}$ and the CCD camera. The output field $\left(E_{\text {out }}(x, y)\right)$ at the the camera plane is

$$
\mathbf{E}_{\text {out }}(x, y)=E_{x}(x, y) h_{x}(x, y) \mathbf{i}+E_{y}(x, y) h_{y}(x, y) \mathbf{j},
$$

where $h_{x}(x, y)$ and $h_{y}(x, y)$ are the computer-generated holograms for the $x$ - and the $y$-component, respectively. 

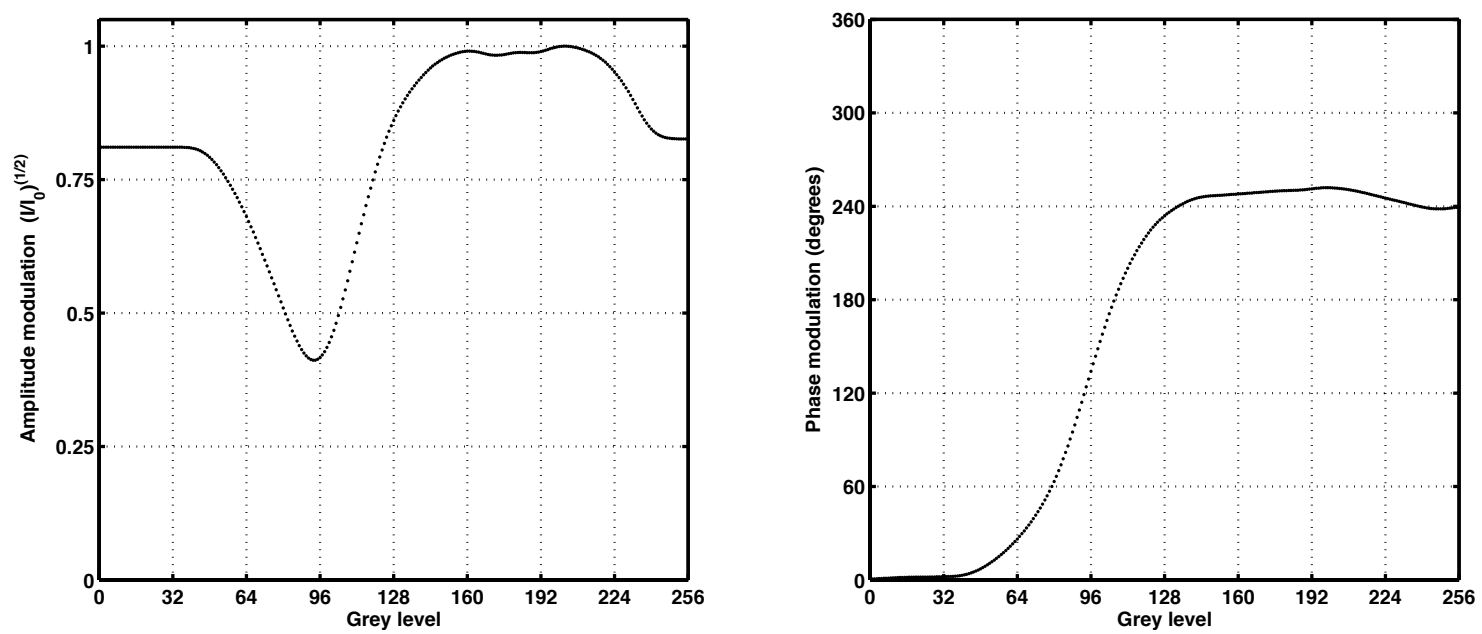

Figure 2: Modulation response for the Holoeye display: (a) amplitude and (b) phase.

Figure 2a show the measured transmittance as a function of the displayed gray level for $\mathrm{SLM}_{1}$; the second one $\left(\mathrm{SLM}_{2}\right)$ performs in a very similar way. It is quite apparent that the amplitude is not constant so no phase-only modulation would be possible using this device. On the other hand, the phase (shown in Figure 2b) presents a nearly linear behavior when the gray level is within the interval 32 to 128 . Note that only phase values from $0^{\circ}$ to $240^{\circ}$ can be reached. The amplitude-phase coupling can be overcome using the codification method explained in the next section.

\section{CODIFICATION PROCEDURE}

Arrizón developed an on-axis holography algorithm for encoding complex optical signals in SLMs with arbitrary amplitude and phase distributions. ${ }^{9}$ This method has been found very suitable for generating ASPB. Now, we briefly summarize the steps required to generate such holograms. Figure 3a shows the set of complex values accessible by $\mathrm{SLM}_{1}$ (Figures $2 \mathrm{a}$ and $2 \mathrm{~b}$ ) using a polar diagram. Let $C_{n m}$ a complex value at position $(n, m)$. If $C_{n m}$ does not belong to the modulation curve $M$, it can be written as the combination of phasors $M_{n m}^{1}, M_{n m}^{2}, E_{n m}^{1}$ and $E_{n m}^{2}$ (see Figure 3a):

$$
\begin{aligned}
& C_{n m}=M_{n m}^{1}-E_{n m}^{1} \quad \text { and } \\
& C_{n m}=M_{n m}^{2}-E_{n m}^{2}
\end{aligned}
$$

where $M_{n m}^{1}$ and $M_{n m}^{2} \in M$. Selecting $M_{n m}^{1}$ and $M_{n m}^{2}$ in such a way that $E_{n m}^{1}=-E_{n m}^{2}$ then

$$
C_{n m}=\left(M_{n m}^{1}+M_{n m}^{2}\right) / 2
$$

Figure $3 \mathrm{~b}$ shows (blue dots) all possible values that can be obtained as a combination of two points belonging to the modulation curve following the condition $E_{n m}^{1}=-E_{n m}^{2}$. Notice that a subset of these values lies within a semicircle of transmittance $T=0.4$ (black line). Moreover, the diameter of this semicircle forms an angle of $30^{\circ}$ relative to the arbitrary phase origin. Full complex modulation can be achieved using a second display, provided that $\mathrm{SLM}_{2}$ performs a similar modulation curve $M$. Fine tuning the optical path of the second arm, the phase origin in $\mathrm{SLM}_{2}$ can be modified and, consequently, if this phase is delayed $180^{\circ}$ with respect to the other display, the system can access any complex value within the circle of transmittance $T=0.4$.

Following the cell-oriented holograms approach, four pixels in the SLM are required to encode each complex value $C_{n m}$, as depicted in Figure 4. It has been demonstrated ${ }^{10}$ that terms $M_{n m}^{1}$ and $M_{n m}^{2}$ are reconstructed on axis whereas the not desired terms $E_{n m}^{1}$ and $E_{n m}^{2}$ are diffracted off-axis; to avoid its contribution, an spatial filter in the back focal plane of lens $L_{1}$ has to be used. 

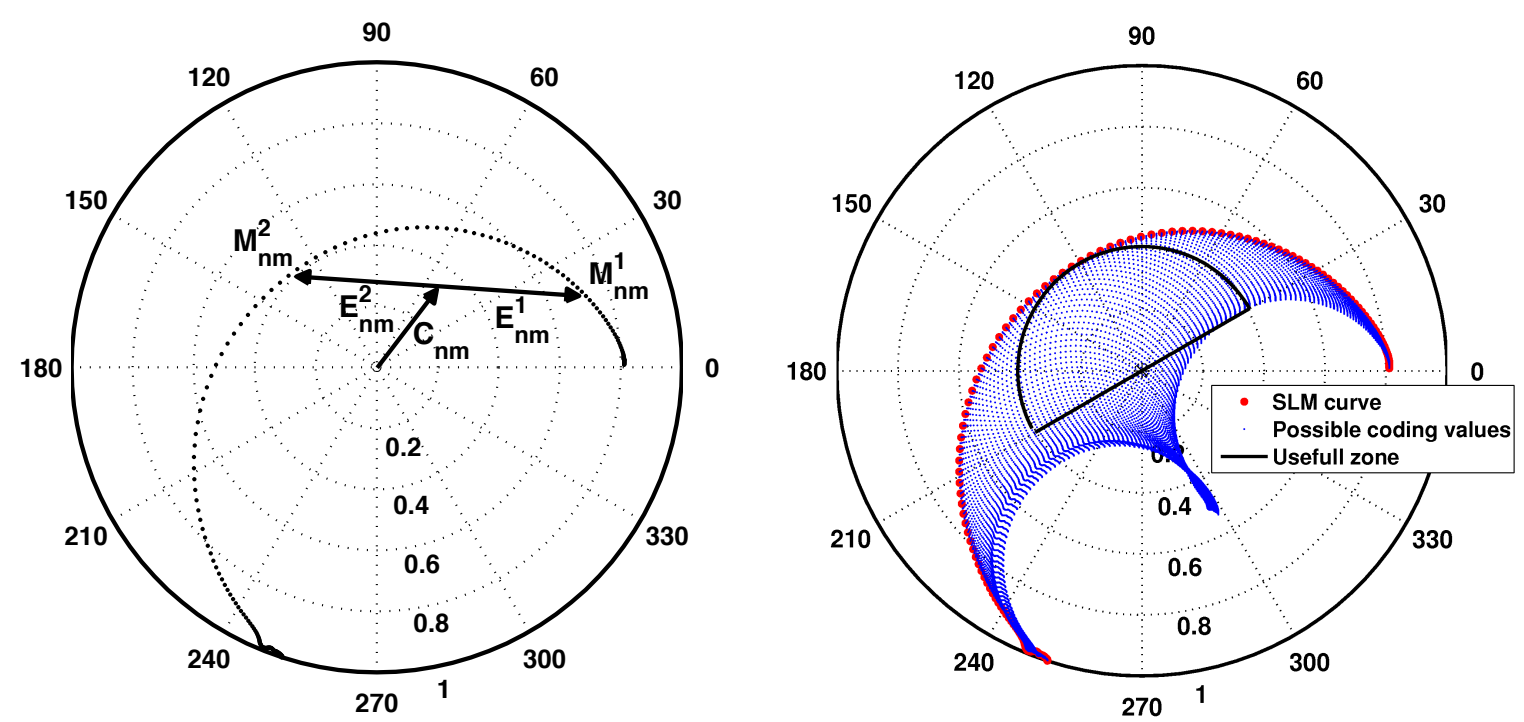

Figure 3: (a) Polar representation of the values accessible by $\mathrm{SLM}_{1}$. (b)Accessible values (in blue) using the codification procedure. The black solid line delimits the useful values.

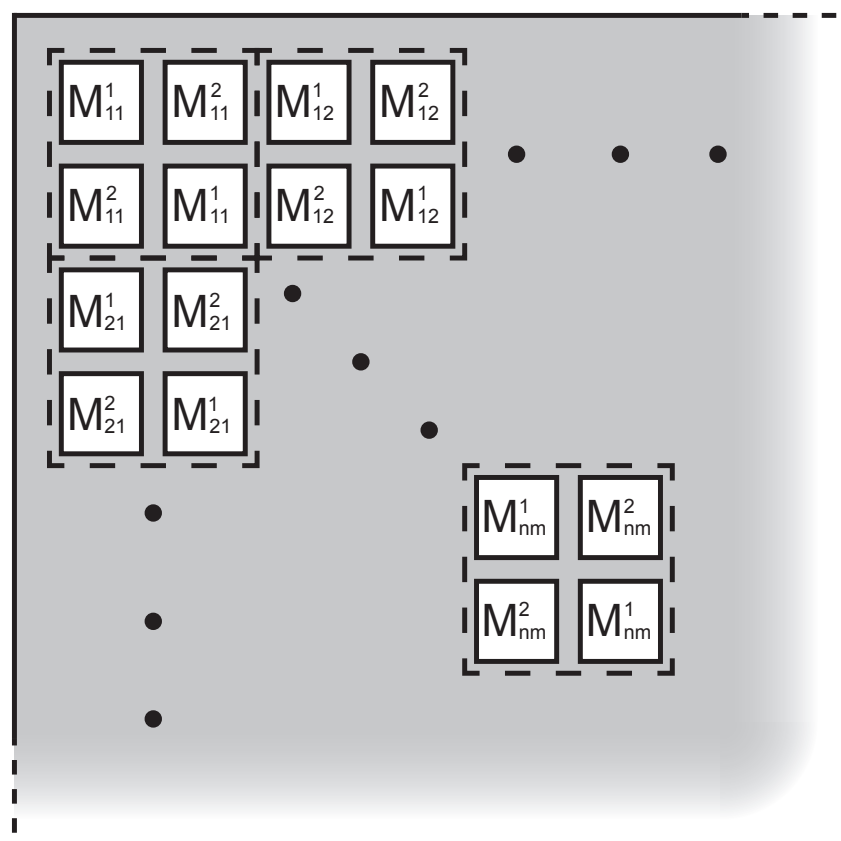

Figure 4: Cell-oriented hologram approach.

\section{NUMERICAL EXPERIMENTS}

Figure 5 show two examples of generalized ASPB beams. On the left, a constant amplitude beam displaying a complicated linearly polarized pattern is shown. The inner mode is radially polarized whereas in the outer mode, the oscillation orientation changes according to $\theta(x, y)=4 \tan ^{-1}(y / x)$. On the right, Figure 5b shows a Hermite-Gauss mode $(2,1)$ with different polarization states in each section of the beam. Figure 6 displays the generated holograms for each display for the two examples considered. Finally, Figure 7 shows the numerical simulation of the irradiance of the output field at the the camera plane $\left(\left|\mathbf{E}_{\text {out }}(x, y)\right|^{2}\right)$ when the orientation of the analyzer is set at different positions $\left(0^{\circ}, 45^{\circ}, 90^{\circ}\right.$ and $\left.135^{\circ}\right)$. 

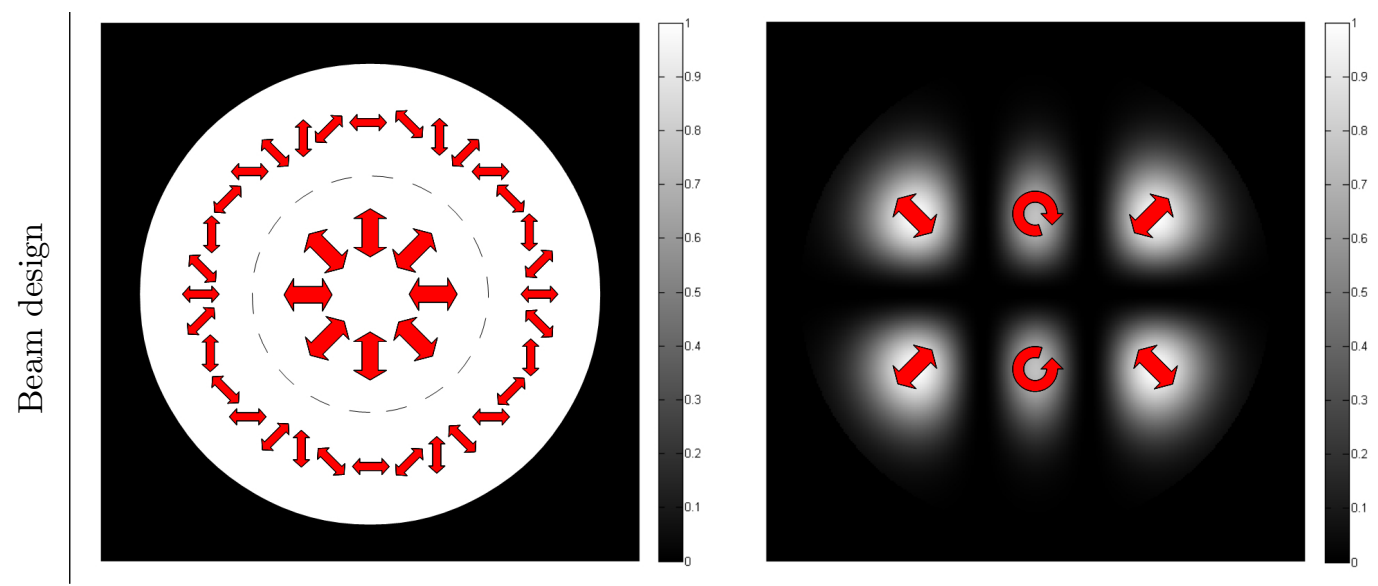

Figure 5: Examples of ASPB intensity patterns: (a) Constant amplitude with radial and star-like polarization, (b) Hermite-Gauss mode $(2,1)$ combining linear and circular polarization.

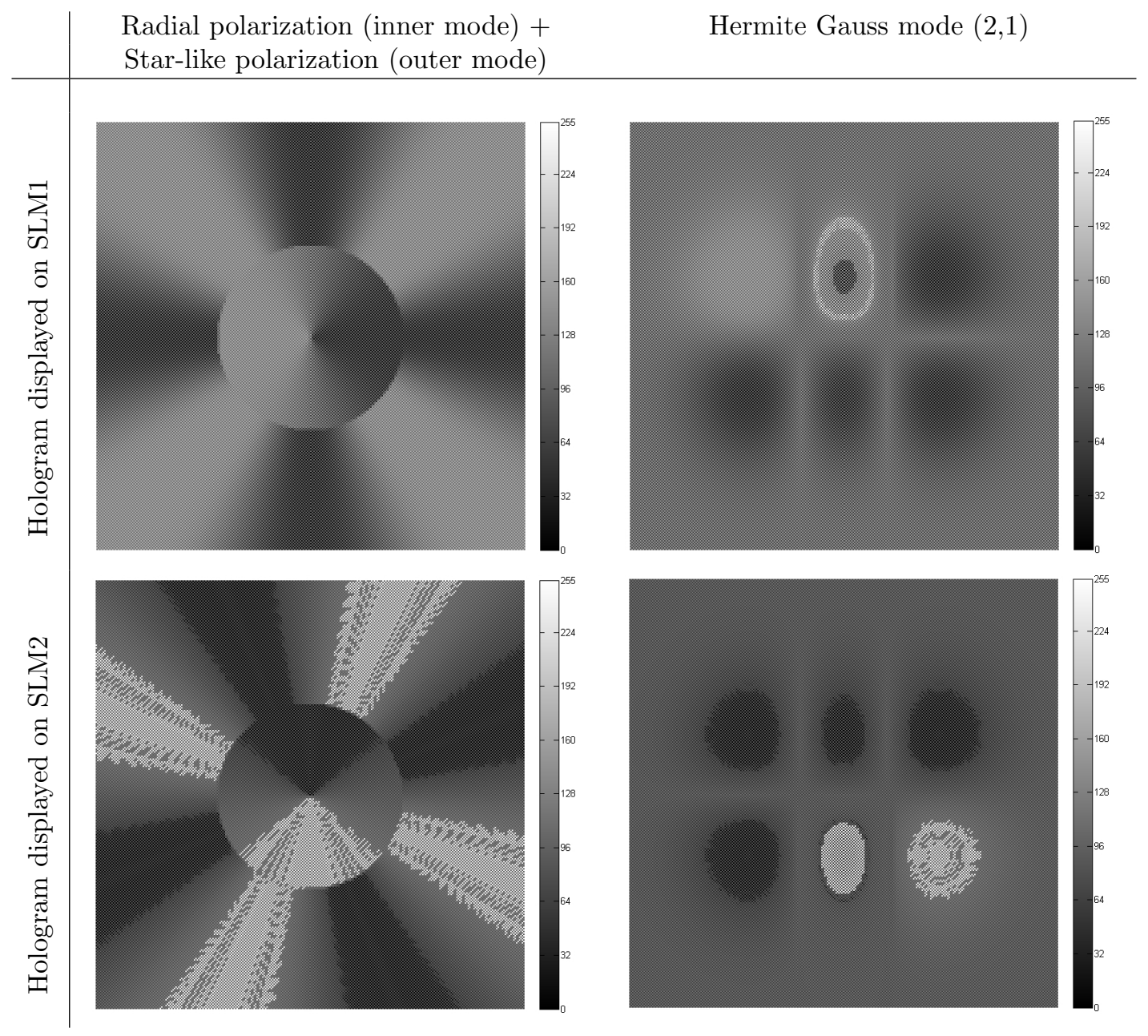

Figure 6: Calculated holograms. 
Radial polarization (inner mode) +

Star-like polarization (outer mode)
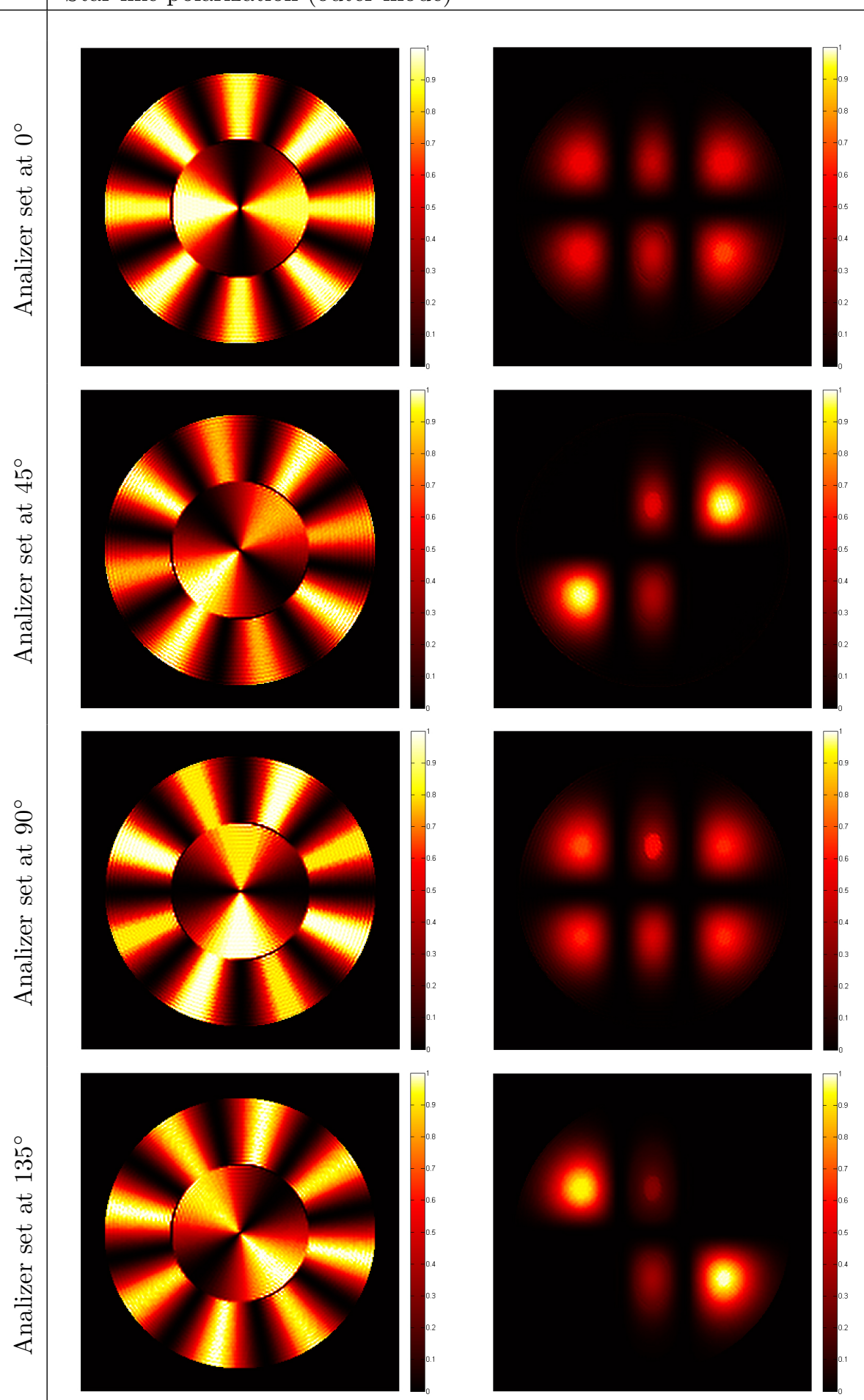

Figure 7: Simulated intensity patterns for different positions of the analyzer. 


\section{CONCLUDING REMARKS}

In this communication we presented a method for generating light beams with controlled polarization and shape using an optical system based on a Mach-Zehnder setup. The transverse components of the beam can be manipulated independently through the use of liquid crystal displays in each arm of the interferometer. Given the limited modulation capabilities of the displays, computer generated holograms have to be used to encode the information. In particular, an adaptation of Arrizón's double-pixel approach was implemented; this method has been demonstrated as very suitable for this kind of problems. Numerical simulations of ASPB with complex polarization distributions and shapes have been carried out demonstrating the feasibility of the proposed technique.

\section{ACKNOWLEDGMENTS}

This paper have been funded in part by the Ministerio de Ciencia e Innovación of Spain, Project FIS2010-17543.

\section{REFERENCES}

[1] Zhan, Q., "Cylindrical vector beams: from mathematical concepts to applications," Advances in Optics and Photonics 1(1), 1-57 (2009).

[2] Davis, J., McNamara, D., Cottrell, D., and Sonehara, T., "Two-dimensional polarization encoding with a phase-only liquid-crystal spatial light modulator," Applied Optics 39(10), 1549-1554 (2000).

[3] Eriksen, R., Mogensen, P., and Glückstad, J., "Elliptical polarisation encoding in two dimensions using phase-only spatial light modulators," Optics Communications 187(4-6), 325-336 (2001).

[4] Maurer, C., Jesacher, A., Fürhapter, S., Bernet, S., and Ritsch-Marte, M., "Tailoring of arbitrary optical vector beams," New Journal of Physics 9, 78 (2007).

[5] Wang, X., Ding, J., Ni, W., Guo, C., and Wang, H., "Generation of arbitrary vector beams with a spatial light modulator and a common path interferometric arrangement," Optics Letters 32(24), 3549-3551 (2007).

[6] Tudela, R., Martin-Badosa, E., Labastida, I., Vallmitjana, S., and Carnicer, A., "Wavefront reconstruction by adding modulation capabilities of two liquid crystal devices," Optical Engineering 43(11), 2650-2657 (2004).

[7] Carnicer, A., Juvells, I., Maluenda, D., Martinez-Herrero, R., Mejías, P., and Schaschek, F., "Generation of arbitrary spatially variant polarized fields using computer generated holograms," Proceedings of the SPIE 8429, 29 (2012).

[8] Arrizón, V., "Optimum on-axis computer-generated hologram encoded into low-resolution phase-modul ation devices," Optics Letters 28(24), 2521-2523 (2003).

[9] Arrizón, V., "Complex modulation with a twisted-nematic liquid-crystal spatial light modulator: doublepixel approach," Optics Letters 28(15), 1359-1361 (2003).

[10] Arrizón, V., González, L., Ponce, R., and Serrano-Heredia, A., "Computer-generated holograms with optimum bandwidths obtained with twisted-nematic liquid-crystal displays," Applied Optics 44(9), 1625-1634 (2005).

[11] Holoeye, "Translucent liquid crystal microdisplays (LCD) http://www.holoeye.com/translucent_LCD_ displays.html." [Online; accessed 8-November-2012]. 